\title{
Maquinado y susceptibilidad a la impregnación de la madera del hule (Hevea brasiliensis)
}

\author{
Victor Rubén Ordóñez Candelaria ${ }^{1}$ \\ José Luis Martínez Castillo ${ }^{1}$
}

\begin{abstract}
RESUMEN
Con una muestra de madera del árbol del hule (Hevea brasiliensis), proveniente de una plantación del estado de Veracruz se efectuó un trabajo de investigación sobre el maquinado y susceptibilidad de impregnación de esta madera, para incrementar el conocimiento de sus propiedades tecnológicas. Los resultados del estudio definen sus características de maquinado de acuerdo con normas ASTM (Regular en casi todos los procesos). Tomando como base estos datos podrán plantearse modificaciones al proceso de maquinado para mejorar el acabado en los casos que así lo requieran. La madera se considera como permeable.
\end{abstract}

PALABRAS CLAVE:

Maquinado de madera, madera del hule (Hevea brasiliensis), penetración, retención, tratabilidad.

\section{ABSTRACT \\ INTRODUCCION}

El árbol del hule (Hevea brasiliensis) es una especie muy importante como productor de látex para Indonesia y
A research work on machining and treatability was carried out with a sample of rubber wood from a plantation in the state of Veracruz, in order to increase the level of technological information on those subjects. The results of the study define the machining characteristics according to ASTM Standards. Taking these results as a basis (regular for most processes), required modifications to the machining process can be acomplished in order to improve the finishing in those cases in which it is required. The rubber wood is considered to be permeable.

KEY WORDS:

Machining, rubber wood (Hevea brasiliensis), penetration, retention, treatability
Malasia, países que cuentan con 4 millones de hectáreas plantadas con esta especie, a nivel mundial existen cerca de 7.5 millones de hectáreas. México tiene plantaciones con 24,721 ha distribuidas en Veracruz, Tabasco y la península de

1 Departamento de Productos Forestales y Conservación de Bosques, Instituto de Ecología, A.C., Apdo. Postal 63, 91000 Xalapa, Ver. México.

Manuscrito recibido para su publicación el 22 de Julio de 1997 
Yucatán (Corella, 1993). Algunas han dejado de producir látex y deberán renovarse, la madera que se obtenga puede utilizarse para fabricar muebles o en la construcción.

Los árboles de Hevea brasiliensis se utilizan principalmente como fuente del látex, que es la materia prima para la fabricación del hule, así como para la elaboración de adhesivos, pinturas para muelles, trajes de buceo, impermeabilizantes, etc. Pero su vida como productor de látex tiene un límite y es entonces cuando las plantaciones deben renovarse. Es conveniente resaltar que la madera de este árbol tiene características de veteado y color que la hacen muy atractiva para la fabricación de muebles, madera para construcción, pisos, de duela, etc. Estas aplicaciones permiten tener un mayor aprovechamiento de este recurso.

Precisamente de una plantación por renovar, localizada en el municipio de Tezonapa, en el estado de Veracruz, se obtuvo una muestra de madera del hule en el aserradero del Campo Experimental "El Palmar", sobre la que se planteó realizar un estudio sobre las características de maquinado y de tratabilidad. El trabajo consistió en estudios de tratabilidad de la madera con preservadores hidrosolubles (en las pruebas se utilizo solamente agua) y la realización de algunas pruebas de maquinado. Las características más importantes de la especie en estudio se presentan a continuación (tomadas de Chudnoff, 1984):

\section{Hevea brasiliensis (plantaciones)}

Familia: Euphorbiaceae

Otros nombres comunes: Arbol de caucho (Venezuela), Sibi-sibi (Guyana), Mapalapa (Surinam), Seringa, Seringuera (Brasil), Capi, Jéve, Shiringa (Perú).
Distribución mundial: Amazonas, ampliamente distribuida en el Sureste de Asia y Oeste de Africa como plantaciones para la producción de hule.

En forma natural puede alcanzar alturas de 30 a $37 \mathrm{~m}$ con fustes cilíndricos largos, con o sin contrafuertes. Cultivado, el árbol puede alcanzar diámetros de hasta $50 \mathrm{~cm}$ generalmente de fustes cortos y con ahusamiento pronunciado.

Características generales de la madera: El duramen presenta un color blancuzco cuando está recién cortado, cambiando a café claro con un tinte rosa, no existe diferencia con la albura. Su textura es moderadamente áspera y uniforme; fibra recta; tiene un olor acre característico y lustre bajo.

Densidad relativa o básica: Determinado como la relación entre peso anhidro y volumen verde varía de 0.46 a 0.52 ; la densidad de la madera seca al aire es de 0.56 a 0.64 .

Propiedades mecánicas: No hay datos disponibles, pero se cita como igual o más resistente que el Pinus sylvestris en todas sus propiedades mecánicas.

Secado y contracciones: De secado rápido al aire, sus alabeos son severos, a menos que se apile con separadores muy cerrados y con carga sobre la pila; deberá secarse bajo cubierta. Requiere de un baño de preservador para controlar la mancha azul y el ataque de taladradores. Los valores de contracciones de verde a anhidro son del $2.3 \%$ en dirección radial y del 5.1 en dirección tangencial.

Propiedades de maquinado: Se reporta como de fácil aserrado, de fácil cepillado con terminado liso; tiende a rajarse en el clavado. 
Durabilidad: Reportada como poco durable y se mancha fácilmente. Muy susceptible al ataque de termitas y taladradores así como los escarabajos destructores de postes (líctidos).

Preservación: Se considera como fácilmente tratable; absorbe $112 \mathrm{~kg} / \mathrm{m}^{3}$ de preservador oleosoluble usando un sistema de baño caliente-frío.

Usos: Se puede utilizar en la construcción en general, tomando las precauciones debidas contra el ataque de termitas y pudrición, para producir pulpa y papel y para fabricación de tableros de fibras y de partículas.

\section{OBJETIVOS Y METAS}

Los objetivos principales de este trabajo son: determinar las características de maquinado de esta madera, cuando se somete a los procesos de cepillado, lijado, barrenado, moldurado y escopleado y evaluar su comportamiento ante el proceso de tratamiento con preservadores hidrosolubles utilizando el sistema de "célula llena".

\section{Metas del maquinado}

Realizar las pruebas de maquinado, con la madera a un contenido de humedad del 12 $\%$ en los siguientes procesos: cepillado, lijado, barrenado, escopleado y moldurado. Determinar la calidad de la superficie maquinada de cada uno de los procesos para caracterizar la madera del hule.

\section{Maquinado}

\section{METODOLOGIA}

La metodología para el trabajo de maquinado, básicamente es la establecida por la norma ASTM D-1666 (ASTM, 1993) con algunas modificaciones como se establecen para barrenado, moldurado, escopleado en el trabajo de Martínez y Martínez-P. (1996) y para cepillado y lijado en Martínez-P. y Martínez 1996. Por ejemplo, el número de probetas: en este trabajo se ensayaron 12 probetas por operación a diferencia de las 50 que establece la norma. Sólo en el caso de lijado y cepillado se redujo la muestra a 11 probetas por desechar una que resultó con defectos. Las probetas se obtuvieron de una muestra de 20 tablas seleccionadas aleatoriamente en el aserradero; en la figura 1 se muestra como se obtuvieron las probetas de maquinado para cada prueba.

La prueba de barrenado se efectuó con una probeta como la que se muestra en la figura 2, haciendo los barrenos en los puntos indicados. Las características de la broca utilizada en esta prueba se observan en la figura $2 b$, que de acuerdo con Martínez y Martínez-P. (1996), proporciona mejores acabados que las otras opciones probadas. En lo referente al equipo se procedió como lo establecen los mismos autores en el trabajo mencionado. En la prueba de cepillado se utilizaron cuchillas con ángulo de corte de 25/ y 30/.

\section{Susceptibilidad a la impregnación}

Las maderas con baja resistencia natural al biodeterioro, requieren de la aplicación de substancias preservadoras para incrementar su vida útil. La aplicación adecuada de estas substancias dependerá de la especie de madera por tratar, el método de tratamiento y el tipo de preservador. Determinar la susceptibilidad de impregnación de la madera del hule permite clasificarla en: permeable, moderadamente resistente, resistente $y$ extremadamente resistente, de acuerdo con lo que presentan Erdoiza y Castillo (1989), utilizando para su clasificación los párametros de retención y penetración. 


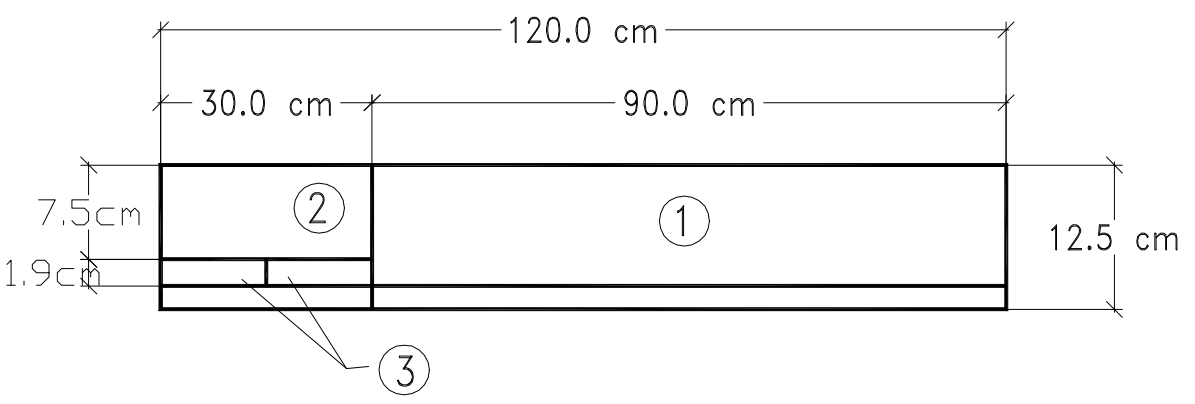

(1) Probetas de cepillado y lijado

(2) Probetas de barrenado, moldurado y escopleado

(3) Probetas de torneado

Figura 1. Patrón de corte de las probetas de maquinado a partir de tablas libres de defectos

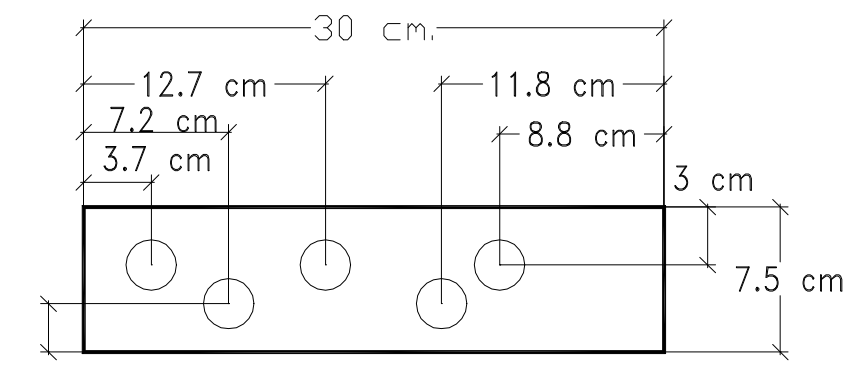

$2.7 \mathrm{~cm}$

a)

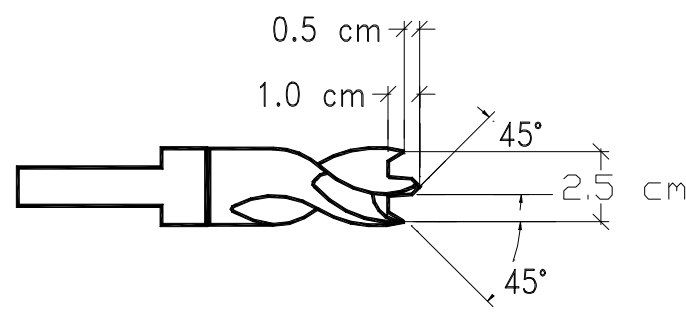

b)

Figura 2. Prueba de barrenado. a) ubicación de las perforaciones b) características del afilado de la broca helicoidal 
La metodología que se aplicó en el estudio es la misma que presentan estos autores y que a grandes rasgos consiste en lo siguiente: Se utilizó madera secada al aire para obtener 30 probetas de $2 \times 2 \times 10 \mathrm{~cm}$. Debido a las características de esta especie no fue posible determinar si la muestra procedía de albura o del duramen. El corte de las probetas se realizó de tal forma que sus planos principales (radial, tangencial y transversal) quedaran orientados con las caras de los prismas. Las probetas se sellaron con resina epóxica por cuatro de sus caras de tal manera que se formaron tres grupos de 10 probetas, en cada uno de los cuales las probetas dejaran expuestas sus caras radial, tangencial o transversal respectivamente. En el proceso de la prueba se colocaron las probetas dentro de los cilindros de una planta piloto de tratamiento y se aplicó un vacío de $20 \mathrm{~mm}$ de mercurio por 30 minutos y después a una presión de $3 \mathrm{~kg} / \mathrm{cm}^{2}$ durante $2 \mathrm{hr}$, utilizando agua como líquido impregnante.

\section{Maquinado}

\section{RESULTADOS}

La calidad del acabado en cada uno de los procesos de maquinado, define el comportamiento de la madera en su transformación. Según la magnitud del defecto o defectos que muestre la superficie maquinada de las probetas se les asigna una de las siguientes calificaciones: Excelente (1), Bueno (2), Regular (3), Pobre (4) y Muy Pobre (5). La forma de identificar cada una de estas calificaciones está definida por la norma ASTM D-1666. Para hacer la clasificación final de la especie en estudio, se utilizan los valores de la Tabla 1, considerando como piezas libres de defectos a las probetas con calificación 1 y 2. El procedimiento consiste en calcular el porcentaje de estas piezas en relación al total de la muestra y con este valor se utiliza para aplicar la Tabla 1.
Tabla 1. Rangos de clasificación final para el maquinado de madera

\begin{tabular}{||l|c|}
\hline \hline CALIFICACION & $\begin{array}{c}\text { PIEZAS LIBRES DE } \\
\text { DEFECTOS (\%) }\end{array}$ \\
\hline Excelente (1) & $90-100$ \\
\hline Bueno (2) & $70-90$ \\
\hline Regular (3) & $50-70$ \\
\hline Pobre (4) & $30-50$ \\
\hline Muy Pobre (5) & $0-30$ \\
\hline
\end{tabular}

Tomando en cuenta los valores de la Tabla 1 y los resultados de las pruebas se procedió a clasificar la madera y en la Tabla 2 se presentan los porcentajes de probetas libres de defectos para cada proceso de maquinado.

\section{Susceptibilidad a la impregnación}

La susceptibilidad de impregnación de una madera queda definida por los índices que se presentan en la Tabla 3, tomada de Erdoiza y Castillo, 1989. Las probetas se cortaron en un plano paralelo a los ensayados, inmediatamente después de la prueba y se le paso un lápiz tinta por todo el corte para observar en donde se teñía de azul por la humedad. Se encontró en todas las probetas de los tres grupos, una penetración total (100\%). En cuanto a los valores de retención se presentan los resultados en la Tabla 4 para cada una de las caras ensayadas. Comparando los valores de penetración y retención para la madera del hule, se clasifica como permeable en cualquier dirección, es decir que el tratamiento de la madera resulta muy eficiente cuando se utiliza un proceso a presión. 
Tabla 2. Resultados de las pruebas de maquinado de la madera del hule (Hevea brasiliensis)

\begin{tabular}{|c|c|c|c|}
\hline PROCESO & PARAMETROS DE LA PRUEBA & \begin{tabular}{|l|} 
PROBETAS SIN \\
DEFECTOS (\%)
\end{tabular} & CLASIFICACION \\
\hline BARRENADO & $\begin{array}{l}\text { Tipo de cuchillas: broca helicoidal } \\
\text { Velocidad: } 1415 \text { rpm } \\
\text { Angulo de corte: afilado en punta de } \\
\text { espuela } \\
\text { Velocidad de avance: manual }\end{array}$ & 50 & REGULAR \\
\hline ESCOPLEADO & $\begin{array}{l}\text { Tipo de cortador: dos filos, } \mathrm{d}=12 \mathrm{~mm} \\
\text { Velocidad de oscilación: } 150 \text { ciclos/min } \\
\text { Angulo de corte: afilado en forma de "X" } \\
\text { en la punta } \\
\text { Velocidad de avance: automático }\end{array}$ & 67 & REGULAR \\
\hline MOLDURADO & $\begin{array}{l}\text { Tipo de cuchillas: para machihembrado } \\
\text { Velocidad: } 7000 \text { rpm } \\
\text { Angulo de corte: } 30^{\circ} \\
\text { Velocidad de avance: manual }\end{array}$ & 83 & BUENO \\
\hline \multirow[t]{2}{*}{ CEPILLADO } & $\begin{array}{l}\text { Tipo de cuchillas: } 4 \\
\text { Velocidad: } 5000 \text { rpm } \\
\text { Angulo de corte: } 25^{\circ} \\
\text { Velocidad de avance: automático }\end{array}$ & 36 & POBRE \\
\hline & $\begin{array}{l}\text { Tipo de cuchillas: } 4 \\
\text { Velocidad: } 5000 \mathrm{rpm} \\
\text { Angulo de corte: } 30^{\circ} \\
\text { Velocidad de avance: automático }\end{array}$ & 36 & POBRE \\
\hline \multirow{3}{*}{ ||LIJADO } & $\begin{array}{l}\text { Tipo de lija: } 100 \\
\text { Dirección del lijado: a favor de la fibra }\end{array}$ & 55 & REGULAR \\
\hline & $\begin{array}{l}\text { Tipo de lija: } 80 \\
\text { Dirección del lijado: a favor de la fibra }\end{array}$ & 64 & REGULAR \\
\hline & $\begin{array}{l}\text { Tipo de lija: } 60 \\
\text { Dirección del lijado: a favor de la fibra }\end{array}$ & 0 & MUY POBRE \\
\hline
\end{tabular}


Tabla 3. Indices de susceptibilidad de impregnación con base en la retención y penetración alcanzada por las muestras de madera utilizando el proceso de "célula llena"

\begin{tabular}{||l|c|c||}
\hline \multicolumn{1}{|c|}{ GRUPO } & $\begin{array}{c}\text { PENETRACION } \\
\text { área penetrada } \\
\%\end{array}$ & $\begin{array}{c}\text { RETENCION \% } \\
\text { (Masa de preservador/masa } \\
\text { de la madera) X 100 }\end{array}$ \\
\hline Permeable & $90-100$ & $75-100$ \\
\hline Moderadamente resistente & $50-89.9$ & $50-74.9$ \\
\hline Resistente & $10-49.9$ & $25-49.9$ \\
\hline Extremadamente resistente & $<10$ & $<25$ \\
\hline
\end{tabular}

Tabla 4. Resultado de las pruebas de impregnación con madera del hule, utilizando el método de "célula llena"

\begin{tabular}{|c|c|c|c|c|c|c|c|c|}
\hline \multicolumn{3}{|c|}{ 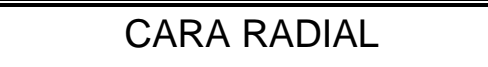 } & \multicolumn{3}{|c|}{ CARA TANGENCIAL } & \multicolumn{3}{|c|}{ "CARA TRANSVERSAL } \\
\hline Probeta & $\underset{\mathrm{gr} / \mathrm{cm}^{3}}{\mathrm{Ra}}$ & Rpe & Probeta & $\underset{\mathrm{gr} / \mathrm{cm}^{3}}{\mathrm{Ra}}$ & Rpe & Probeta & $\underset{\mathrm{gr} / \mathrm{cm}^{3}}{\mathrm{Ra}}$ & Rpe \\
\hline $1 \mathrm{R}$ & 0.49 & 0.82 & $1 \mathrm{~T}$ & 0.50 & 0.84 & $1 \mathrm{TR}$ & 0.55 & 0.92 \\
\hline $2 \mathrm{R}$ & 0.51 & 0.86 & $2 \mathrm{~T}$ & 0.51 & 0.86 & 2TR & 0.53 & 0.89 \\
\hline $3 R$ & 0.44 & 0.74 & $3 T$ & 0.50 & 0.84 & 3TR & 0.44 & 0.74 \\
\hline $4 \mathrm{R}$ & 0.50 & 0.84 & $4 \mathrm{~T}$ & 0.50 & 0.84 & $4 T R$ & 0.50 & 0.84 \\
\hline $5 R$ & 0.48 & 0.81 & $5 \mathrm{~T}$ & 0.52 & 0.87 & 5TR & 0.50 & 0.84 \\
\hline $6 \mathrm{R}$ & 0.45 & 0.76 & $6 \mathrm{~T}$ & 0.52 & 0.87 & $6 \mathrm{TR}$ & 0.53 & 0.89 \\
\hline $7 \mathrm{R}$ & 0.49 & 0.82 & $7 \mathrm{~T}$ & 0.53 & 0.89 & 7TR & 0.50 & 0.84 \\
\hline $8 \mathrm{R}$ & 0.47 & 0.79 & $8 \mathrm{~T}$ & 0.51 & 0.86 & $8 T R$ & 0.55 & 0.92 \\
\hline $9 R$ & 0.46 & 0.77 & $9 \mathrm{~T}$ & 0.54 & 0.91 & 9TR & 0.53 & 0.89 \\
\hline $10 \mathrm{R}$ & 0.54 & 0.91 & $10 \mathrm{~T}$ & 0.58 & 0.97 & 10TR & 0.55 & 0.92 \\
\hline \multicolumn{2}{|l|}{ Promedio } & 0.812 & \multicolumn{2}{|l|}{ Promedio } & 0.875 & \multicolumn{2}{|l|}{ Promedio } & 0.869 \\
\hline
\end{tabular}

Ra: $\quad$ Retención de líquido por volumen de madera

Rpe: $\quad$ Retención de líquido con relación al peso de la madera, utilizando un valor de la densidad relativa, $\mathrm{DA}=0.596$ (peso anhidro/volumen anhidro) calculado con base en el valor de $D R=0.51$ (peso anhidro/volumen verde) (FPL, 1987). 


\section{DISCUSION Y CONCLUSIONES}

Con base en los resultados de las pruebas de maquinado se puede establecer que la madera del hule presenta acabados de calidad regular, en la mayor parte de los procesos; solamente el cepillado presenta acabados pobres aunque faltaría saber si el ángulo de 35o mejoraría la superficie maquinada. De la misma manera, con la lija de grado 60 las caras presentan un acabo muy pobre con "grano" arrancado en todas las probetas.

Aunque la clasificación general de la madera es aceptable, se deben plantear estudios posteriores con la finalidad de mejorar los acabados, para lo que se deberá estudiar la madera modificando las variables de los procesos, en los que sea posible hacerlo, como serían velocidades de alimentación, ángulos de corte, velocidad de corte, etc.

La madera del hule es de fácil impregnación por medios a presión. Aún con sistemas de baño caliente-baño frío se obtienen buenos resultados. Esto permite usar la madera en condiciones adversas a pesar de su baja durabilidad, si se aplica el preservador adecuado.

\section{RECONOCIMIENTOS}

Este trabajo fue realizado con fondos fiscales del Departamento de Productos Forestales y Conservación de Bosques del Instituto de Ecología, A.C. con la clave 90213.

\section{REFERENCIAS}

American Society for Testing and Materials. (ASTM). 1993. Standard Methods for Conducting Machining Tests of Wood and Wood-base Materials. D-1666-87. Annual Book of ASTM Standards
Volume 04.09 Wood. Philadelphia, p:257-276.

Chudnoff, M. 1984. Tropical timbers of the world. Agriculture Handbook 607. Washington, D.C., U.S. Department of Agriculture. Forest Service. 466p.

Corella J., F. y A. Sol S. 1993. Establecimiento de 2 hectáreas de un banco de germoplasma de hule (Hevea brasiliensis Muell Arg.). Sexta reunión científica, forestal y agropecuaria. Centro de Investigaciones Forestales y Agropecuarias de Tabasco. Publicación especial № 6 .

Erdoiza S., J. e I. Castillo M. 1989. Susceptibilidad de impregnación con preservadores de cincuenta especies maderables mexicanas. La madera y su uso No. 22. Instituto de Ecología, A.C. Universidad Autónoma Metropolitana. Xalapa, México. 18p

Forest Products Laboratory. 1987. Wood Handobook: Wood as an engineering material. Agriculture Handbook 72. U.S. Department of Agriculture Washington D.C. $466 p$.

Hoadley, R.B. 1980. Understanding Wood. A Crafstman's Guide to Wood Technology. The Taunton Press. Newton Conn. p:145-163.

Longwood, F.R. 1961. Puerto Rican Woods. Their machining, seasoning and related characteristics. USDA Forest Service, Agriculture Handbook No. 205. Washington D.C. p:145-160.

Martínez C., J.L. y E. Martínez-P.C. 1996. Características de maquinado de 32 especies de madera. Madera y Bosques (2) 1:45-62. 
Martínez-P. C., E y J.L. Martínez C. 1996. Características de cepillado y lijado de 33 especies de madera. Madera y Bosques (2)2:11-27.

Secretaría de Agricultura y Recursos Hidráulicos (SARH). 1983. Estudio promocional de 43 Especies Forestales Tropicales Mexicanas. Tomo 1. Reporte Técnico. Programa de cooperación científica y Técnica México-Yugoslavia. Subsecretaría Forestal. Secretaría de Agricultura y Recursos Hidráulicos. México D.F. 